\title{
Synthesis and characterization of azine-functionalized zinc cation metal- organic frameworks nanostructures upon silk fibers under ultrasound irradiation, study of pores effect on morphine adsorption affinity
}

\author{
Amir Reza Abbasi *a , Mohammadreza Yousefshahi ${ }^{\text {a }}$, Azadeh Azadbakht ${ }^{\text {b }}$ \\ ${ }^{a}$ Faculty of Chemistry, Razi University, 67194, Kermanshah, Islamic Republic of Iran. \\ Tel. and Fax: +98 83 34277464, E-mail address: ar.abbasi@ razi.ac.ir \\ ${ }^{\mathrm{b}}$ Department of Chemistry, Faculty of Science, Khorramabad Branch, Islamic Azad \\ University, Khorramabad, Islamic Republic of Iran.
}

\begin{abstract}
Thin films of a three-dimensional porous $\mathrm{Zn}(\mathrm{II})-\mathrm{MOFs}$ (TMU-4 and TMU-5) were deposited on surfaces of silk fiber via a stepwise manner under ultrasound irradiation. The Zn(II)-MOFs@fibers were studied for removal and extraction of morphine from aqueous samples and the effect of host-guest interaction on adsorption and desorption efficiency were evaluated. The effect of sequential dipping steps in growth of $\mathrm{Zn}(\mathrm{II})-\mathrm{MOFs} @$ fiber has been studied. These systems depicted a decrease in the size accompanying a decrease in the sequential dipping steps.
\end{abstract}

Keywords: Ultrasound irradiation; Nano; Metal-organic framework; Morphine.

\section{Introduction}

The field of metal-organic frameworks (MOFs), which are also called porous coordination polymers (PCPs), has been growing tremendously over the last two decades [1,2]. This fascinating class of crystalline hybrid materials, which are formed by association of metal centers or clusters linked by organic ligands, offer an unique chemical 
versatility combined with a designable framework and an unprecedentedly large and permanent porosity [3-5]. These materials have enormous potential for many practical structure-related applications. This includes the more traditional areas of storage, separation or controlled release of gases, catalysis, sensing, and drug delivery, as well as the adsorptive removal of hazardous materials, which are based on the pore size and shape as well as the host-guest interactions involved [6-11]. However, the majority of these applications are based on the ability of MOFs to behave as hosts for certain molecules. MOFs have already been tested as micro/nanoporous materials with exceptionally high porosity, uniform but tunable pore size, and with well-defined molecular adsorption sites [12]. Apart from their use as bulk materials; these frameworks could be processed as supported homogeneous porous thin films on various surfaces. Controlling the assembly of metal-organic frameworks thin films on different substrates is currently recognized as one of the most important issues in the synthesis of functional materials [12,13]. Different strategies have been developed in the literature to fabricate thin films of MOFs. These technical approaches can be grouped in several ways such as surface functionalization [14], layer-by-layer (LBL) [15] and electrospun nanofibrous filters [16].

In this work we report the layer-by-layer deposition of a microporous $\mathrm{Zn}(\mathrm{II})$-MOF material on the surface of natural silk fiber with $-\mathrm{COOH}$ surface functionalization under sonication. The advantage of ultrasonic method is the homogeneous coating of small structures [17]. The Zn(II)-MOFs TMU-4@silk fiber and TMU-5@silk fiber $($ TMU-4 = $\left[\mathrm{Zn}_{2}(\mathrm{oba})_{2}(4-\mathrm{bpdb})\right] \cdot 3 \mathrm{DMF}$ and TMU-5 $\left.=\left[\mathrm{Zn}_{2}(\mathrm{oba})_{2}(4-\mathrm{bpdh})\right] \cdot 3 \mathrm{DMF}\right)$ have been investigated for applications in loading and release of morphine molecules. TMU-4 shows 1D, large pores with apertures of about $5.3 \times 9 \AA$, respectively, whereas TMU-5 shows $3 \mathrm{D}$, interconnected, narrow pores with an aperture of about $4.4 \times 6.2 \AA$ [18]. Morphine is an analgesic opioid and is one of the most potent pain relievers. It has been used for pain 
relief and other indications for years. Morphine is highly addictive and its use is associated with significant physical and psychological dependence. Morphine has a high potential for addiction; tolerance and psychological dependence develop rapidly, although physiological dependence may take several months to develop.

The deposition of MOF thin films on flexible polymer surfaces might be a new path for the fabrication of functional materials for different applications, such as protection layers for working clothes, gas separation and thin-film drug delivery materials in the textile industry [14]. In anchoring MOFs to surfaces (SURMOFs), the first step is the functionalization of substrate or self-assembled monolayers (SAMs) [19] and the second step is the growth of MOF. Deposition of microcrystalline MOF at alumina [20,21], silica [20] and on surfaces of flexible organic polymers [22] were reported, but in this work we used silk fibers as substrate, thus due to existence of $-\mathrm{COOH}$ groups on the surface of these silk fibers no SAM formation was required and in a very simple and effective procedure at ambient pressure and temperature, $\mathrm{Zn}(\mathrm{II})$-MOF coating of the silk fibers were done successfully by LBL technique.

\section{Experimental section}

\subsection{Materials and physical techniques}

All reagents and solvents were used as supplied by Merck Chemical Company and used without further purification. The silk fiber was obtained from Guilan Silk Company. The natural silk fibers were pre washed using an aqueous solution containing $\mathrm{NaOH}(\mathrm{pH}=9)$, at $25{ }^{\circ} \mathrm{C}$ for $5 \mathrm{~min}$, followed by washed several times with water and dried at ambient temperature. X-ray powder diffraction (XRPD) measurements were done on a Philips X'pert diffractometer with monochromatic $\mathrm{Cu} \mathrm{K} \alpha$ radiation. The simulated XRD powder pattern based on single crystal data were prepared using Mercury software [23]. The 
Brunauer-Emmett-Teller (BET) analyses were recorded on Belsorp-mini II japan, and used to determine the specific surface area, pore size and volume. The samples were characterized with a scanning electron microscope (SEM, Philips XL 30 and S-4160) with gold coating. Ultrasonic generators were carried out on a Eurosonic 4D (continuous mode, output power: $350 \mathrm{~W}$ ). Ultrasonic generators have water circulation system and double jacketed vessel. The effects of sonication in growth of the $\mathrm{Zn}(\mathrm{II})$-MOFs upon fiber were studied in $350 \mathrm{~W}$. The average particle sizes were prepared using Microstructure measurement software. In situ UV/vis spectrum experiment has been carried out on a PG Instruments, T80 + UV/vis/NIR spectrophotometer within the wavelength range 190-800 $\mathrm{nm}$, using the same solvent in the examined solution as a blank. Infrared spectra were taken with a FT-IR Bruker, vector 22 spectrometer using KBr pellets in the $400-4000 \mathrm{~cm}^{-1}$ range.

\subsection{Synthesis of TMU-4@ silk and TMU-5@silk surfaces}

The ligand 1,4-bis(4-pyridyl)-2,3-diaza-1,3-butadiene (4-bpdb) was synthesized according to previously reported methods [24]. Before the experiment began, silk fibers were immersed in an alkaline solution. In alkaline $\mathrm{pH}$, the surface of fiber becomes negatively charged due to deprotonation of the carboxylic group present at the fiber's surface. The growth of TMU-4@silk fiber was achieved by sequential dipping in alternating bath of aqueous $\mathrm{Zn}\left(\mathrm{NO}_{3}\right)_{2} \cdot 6 \mathrm{H}_{2} \mathrm{O}(0.189 \mathrm{~g}, 0.64 \mathrm{mmol})$ and a DMF solution of 4-bpdb $(0.238 \mathrm{~g}, 1 \mathrm{mmol})$ and 4,4'-oxybisbenzoic acid $\left(\mathrm{H}_{2} \mathrm{Oba}\right)(0.254 \mathrm{~g}, 1 \mathrm{mmol})$ under ultrasound bath. TMU-5@silk were obtained using the same reaction conditions and ratios as used for the isolation of TMU-4, but using 4-bpdh (4-bpdh = 2,5-bis(4-pyridyl)-3,4diaza-2,4-hexadiene) instead of 4-bpdb (Figure 1). The first layer was fabricated by immersing the silk- $\mathrm{COO}^{-}$surface into an solution of $\mathrm{Zn}(\mathrm{II})$ and then in solution of donor 
ligands (1 cycle). When negative fiber was immersed in an aqueous solution of zinc(II) nitrate, $\mathrm{Zn}(\mathrm{II})$ ions are attracted to the fiber surface. The dipping step in solution of donor ligands allowed the formation of TMU-4 and initiated the formation of new TMU-4 particles, as illustrated in Figure 1. The thickness of the multilayers was increased with the increase of the deposition cycles. The dipping step in each $\mathrm{Zn}$ (II) and donor ligands solutions was $1.5 \mathrm{~min}$ followed by some rinses in pure DMF each for $1 \mathrm{~min}$. In order to investigate the deposition of the first 15 MOF layers on the surface of the silk fiber the substrate was dipped alternatively into SBU- and linker solution with washing with DMF in between.

\section{Results and discussion}

3.1 Fourier transform infrared spectroscopy (FT-IR) and X-ray powder diffraction (XRPD)

Dense coatings of $\mathrm{Zn}(\mathrm{II})-\mathrm{MOF}$ have been synthesized on silk fibers from layer-by-layer growth from molecular precursors (Figure 1).

\section{Suggested position for Fig. 1}

Due to the small amount and thickness of the deposited MOF film, the standard technique of FT-IR is suited for the investigation of film quality. Therefore, after each deposition cycle an absorption spectrum of the dried substrate was recorded. The increases of the intensity of the vibrational bands from TMU-4 and TMU-5 phase are proportional to the number of performed deposition cycles. It can be concluded that the characterization of MOF films with a low number of performed deposition cycles can be better achieved using the FT-IR spectra than XRPD, due to its limitation in detection of films with a thickness $<40 \mathrm{~nm}[25]$. The change of the intensity was observed after the first deposition cycle on the silk surface. Although the observed change of the intensity is small, the 
intensity increases continuously with each further deposition cycle (Figure S1 (Supporting Information)). The linear increase of the absorbance indicates a regular assembly of the cationic and anionic building blocks. FT-IR data for TMU-4@silk (KBr pellet, v/ $\left.\mathrm{cm}^{-1}\right)$ selected bands: 659(m), 776(m), 1089(m), 1159(s), 1241(vs), 1412(vs), 1500(m), 1608(vs), 1679(s), 2927(m) and 3417(w-br). FT-IR data for TMU-5@silk (KBr pellet, $\left.v / \mathrm{cm}^{-1}\right)$-selected bands: 658(m), 777(m), 1091(m), 1159(s), 1238(vs), 1408(vs), 1501(m), 1608(vs), 1677(s), 2927(m), 2927(m) and 3428(w-br).

To determine the crystal phase of Zn(II)-MOF (TMU-4 and TMU-5) formed upon silk fiber, X-ray powder diffraction (XRPD) measurements were carried out over the diffraction angle $(2 \theta)$ of $0-40^{\circ}$. Figure S2 (Supporting Information) (left) shows the XRPD patterns; simulated from single crystal X-ray data of TMU-4 (A), as-synthesized TMU-4 (B), pristine silk fibers (C) and TMU-4@silk after applying 15 deposition cycles (D). Figure S2,E (Supporting Information) (left) shows the X-Ray powder diffraction patterns of TMU-4@silk soaked in an aqueous solution $\left(10^{-4} \mathrm{~mol} \mathrm{~L}^{-1}\right)$ of morphine at room temperature for 3 days. It is worth noting that when the silk fibers were soaked in the solution of starting materials, most of the peaks in the XRPD data distinctly weakened. Our results show clearly that the structure changed during coating of TMU-4 on the silk fibers. The obtained pattern in Figure S2,B (Supporting Information) (left) match with the pattern of monoclinic TMU-4, space groups $P 2{ }_{1} / c$ with the lattice parameters $a=$ 12.5460(4) $\AA, b=26.6330(7) \AA, c=15.7340(6) \AA$ and $z=4$ [18]. Figure S2 (Supporting Information) (right) shows the XRPD patterns; simulated from single crystal X-ray data of TMU-5 (A), as-synthesized TMU-5 (B), pristine silk fibers (C) and TMU-5@silk after applying 15 deposition cycles (D). This figure shows the X-Ray powder diffraction patterns of TMU-5@silk soaked in an aqueous solution $\left(10^{-4} \mathrm{~mol} \mathrm{~L}^{-1}\right)$ of morphine at room 
temperature for 3 days. The eight major peaks found at $6.70^{\circ}, 7.70^{\circ}, 9.35^{\circ}, 10.95^{\circ}, 12.20^{\circ}$, $14.70^{\circ}, 14.77^{\circ}$ and $15.94^{\circ}$ on the 2 theta scale correspond respectively to the (200), (002), (202) $),(202),(111),\left(112^{-}\right),(310)$ and (312) crystal planes. The obtained pattern match with the pattern of monoclinic TMU-5, space groups $C 2 / c$ with the lattice parameters $a=$ 26.8080(14) $\AA, b=8.1923(5) \AA, c=23.3025(16) \AA$ and $z=8$ [18]. Acceptable matches with slight difference in $2 \theta$, were observed between the simulated XRPD pattern and the experimental data [18]. The results indicated that $\mathrm{Zn}(\mathrm{II})$-based MOF formed on the silk fiber (Figure S2 (Supporting Information)) and the thickness of the coated Zn(II)-MOF films were increased by increasing the cycles of layer by layer coating of TMU-4 and TMU-5 on the silk fibers (Figure S1 (Supporting Information)) [17], the wide peak at 17$23^{\circ}$ corresponds to the silk substrate $[17,26]$.

\subsection{Effects of sequential dipping steps}

Particle sizes and morphology of nanoparticles are depending on sequential dipping [27]. Effect of different sequential dipping in growth of $\mathrm{Zn}(\mathrm{II})$-MOFs upon fiber were studied at $\mathrm{pH}=9$. The results suggest that with increasing in the fiber dipping steps into SBU- and linker solution, growth takes place on more nuclei, the $\mathrm{Zn}^{2+}$ and donor ligands (4-bpdh/4-bpdh and $\mathrm{H}_{2} \mathrm{Oba}$ ) attraction increases, and subsequently the concentration and size of $\mathrm{Zn}(\mathrm{II})-\mathrm{MOF}$ particles upon silk fiber increases [28-30]. For the sake of investigating the morphology of the prepared coating samples, the SEM images of samples were studied. The SEM images of the non-modified natural fiber were compared with images after applying 4 and 15 deposition cycles of TMU-5 upon silk fiber (Figure 2A-C). The surface of the individual fibers is covered by a continuous film of separated crystals with an average size of 207 and $310 \mathrm{~nm}$ for 4 and 15 deposition cycles, respectively. However, the SEM images with a low magnification also exhibit areas with 
big agglomerates of the deposited MOF. This can be understood by considering the possible storage effect of the unreacted material between the fibers. These facts are repeated for TMU-4 upon silk fiber (Figure 2D and E).

\section{Suggested position for Fig. 2}

\subsection{Ultrasound effects}

In order to investigate the role of sonicating on the nature of products, blank reaction was performed without ultrasound irradiation. In this reaction, TMU-5 particles on silk fibers were prepared by sequential dipping steps without sonicating. The average particle size for ultrasound method is around $207 \mathrm{~nm}$ (Figure 2B) while, the average particle size for blank sample (Figure 3) in similar conditions is over $284 \mathrm{~nm}$. Results show that in present of ultrasound radiation, particle sizes are in a low range. This finding has already been observed in other studies on ultrasound-assisted synthesis of nano-particles [26]. The sonochemical irradiation of a liquid causes two primary effects, namely, cavitations (bubble formation, growth, collapse) and heating. When the microscopic cavitations bubbles collapse near the surface of the solid substrate, they generate powerful shock waves and microjets that cause effective stirring/mixing of the adjusted layer of liquid. The after-effects of the cavitations are several hundred times greater in heterogeneous systems than in homogeneous systems [27]. In our case, the ultrasonic waves promote the fast migration of the newly-formed nanoparticles to the fabric's surface. There is reliable evidence that applying ultrasound not only induces nucleation, but also increases reproducibility. Another effect of ultrasound on nucleation is shortening the induction time between the establishment of supersaturation and the onset of nucleation and crystallization. The cavitation events allow the excitation energy barriers associated with nucleation to be surmounted, in which case it should be possible to correlate the number of cavitation and nucleation events in a quantitative way [31]. It has been suggested that 
nucleation caused by scratching the walls of a vessel containing a supersaturated solution with a glass rod spatula could be the result of cavitation [27].

\section{Suggested position for Fig. 3}

\subsection{Adsorption affinity}

The porosity of MOF films deposited on substrate surfaces is an important point concerning the possible use of such functional materials for different purposes. TMU-4 has one-dimensional open channels (aperture size of $5.3 \times 9 \AA$ ) running along the (101) direction in which the internal surface is decorated by the azine groups of the 4-bpdb ligands. TMU-5 shows narrow, three-dimensional interconnected pores (aperture size of $4.4 \times 6.2 \AA$ ) that are also functionalized with azine groups (Figure 1B). Surface area of TMU-4 and TMU-5 were determined by $\mathrm{N}_{2}$ adsorption. A type-II $\mathrm{N}_{2}$ isotherm collected at $77 \mathrm{~K}$ and 1 bar on the conventional heating synthesized TMU-4 revealed that $\mathrm{N}_{2}$ molecules could not diffuse within its pores under these conditions. Surprisingly, even though TMU-5 has narrower pores than TMU-4, we found that it is porous to $\mathrm{N}_{2}(144.49$ $\mathrm{cm}^{3} / \mathrm{g}$ at 1 bar; BET surface area: $582.4 \mathrm{~m}^{2} / \mathrm{g}$ ) at $77 \mathrm{~K}$, for which it showed reversible type-I isotherms (Figure S3 (Supporting Information)). For augment of porosity in Zn(II)MOF@silk, we successfully tested its porosity with guest molecules by suspending it in an aqueous solution of morphine. We tested for the amount of morphine that can be inserted in the pores. The guest content was estimated by XRPD (Figure S2 (Supporting Information)) and UV/vis spectroscopy (Figure 4) [32]. The change of intensity and width indicates that the resulting solid Zn(II)-MOF@silk retains the host framework crystallinity as morphine molecules diffused in. To explore the absorption ability of the $\mathrm{Zn}$ (II)-MOF to morphine, fresh sample of $\mathrm{Zn}(\mathrm{II})-\mathrm{MOF}(1.3 \mathrm{mg})$ was immersed in an aqueous $(50 \mathrm{~mL})$ solution of morphine $(0.0245 \mathrm{mmol})$ and were monitored in real time with UV/vis spectroscopy. Adsorption of guest was spontaneous and endothermic, and the entropy (the 
driving force of the adsorption) increases with the adsorption of guest. Entropic hydrophobic interactions occur when a guest replaces the water within a cavity. An increase in entropy increases the favorability of the process. The sorption capacity of $\mathrm{Zn}$ (II)-MOF increases with the increase of contact time (Figure 4). The rapid decline of the UV/vis peak also proves that TMU-5 can absorb morphine higher than that of TMU-4. As shown in Figure 4, best results were obtained when TMU-5 was used as a host framework. Increasing the basicity of $\mathrm{N}$-donor ligands leads to improvement in absorption activity. Results show that among the two compounds, TMU-5 has the higher absorption activity upon increasing its basicity of azine function in the $\mathrm{N}$-donor ligand. The lower sorption for TMU-4 is attributed to the pore size and honeycomb framework. In TMU-4, a three-dimensional honeycomb framework with double interpenetration was obtained by coordination of the $\mathrm{oba}^{2-}$ and 4-bpdb ligands to $\mathrm{Zn}(\mathrm{II})$ nodes.

\section{Suggested position for Fig. 4}

\subsection{Release assays}

The encapsulated guest could be easily removed from the frameworks upon immersion of guest@MOFs in organic solvents. To further investigate the kinetics of the guest delivery of the framework, UV/vis spectra were recorded at room temperature. The temporal evolution UV/vis spectrum for morphine in ethanol solution becomes stronger with increasing morphine content (Figure 5). The delivery of morphine in ethanol increases with time, indicating that the morphine release is governed by the host-guest interaction. The TMU-5-morphine interaction is stronger than TMU-4-morphine interaction, so the delivery of morphine from TMU-4 can be faster than TMU-5 (Figure 6). The exceptional affinity of TMU-5 for morphine may be attributed to the basicity of $\mathrm{N}$-donor ligand and structural character of the three-dimensional interconnected pores made of 4-bpdh. Once these interactions fade out with increasing morphine extrusion, the 
delivery in the second stage is mainly governed by a free diffusion process and a complete morphine release from TMU-5 needs more than 14 days to attain the equilibrium state. However, morphine release from TMU-4 occurs within 12 days and then moves toward the equilibrium state (Figure 6).

\section{Suggested position for Figs. 5 and 6}

\section{Conclusions}

In summary, we reported the fabrication of $\left[\mathrm{Zn}_{2}\left(\mathrm{H}_{2} \mathrm{Oba}\right)_{2}(4-\mathrm{bpdb})\right] \cdot 3 \mathrm{DMF}(\mathbf{T M U}-4)$ and $\left[\mathrm{Zn}_{2}\left(\mathrm{H}_{2} \mathrm{Oba}\right)_{2}(4-\mathrm{bpdh})\right] \cdot 3 \mathrm{DMF}$ (TMU-5) metal-organic frameworks nanostructures upon silk fiber using layer-by-layer method at ambient pressure and temperature. Due to existence of - $\mathrm{COOH}$ groups on the surface of the silk fibers no self-assembled monolayer formation was required. The effect of sequential dipping steps in growth of $\mathrm{Zn}$ (II)MOFs@fiber have been studied. These systems depicted a decrease in the size accompanying a decrease in the sequential dipping steps. XRPD analyses indicated that the prepared $\mathrm{Zn}(\mathrm{II})-\mathrm{MOF}$ on silk fibers were crystalline. The deposition of MOF thin films on natural fiber surfaces might be a new path for the fabrication of functional materials for different applications, such as protection layers for working clothes, thin films on flexible polymer surfaces and gas separation materials in the textile industry. $\mathrm{Zn}(\mathrm{II})-\mathrm{MOF}$ upon silk may indeed is suitable for applications requiring frequent loading and unloading of morphine molecules.

\section{Acknowledgements}

Support of this investigation by Iran National Science Foundation: INSF and Razi University of Kermanshah are gratefully acknowledged. 


\section{References}

[1] F.A. Almeida Paz, J. Klinowski, S.M.F. Vilela, J.P.C. Tome, J.A.S. Cavaleiro, J. Rocha, Ligand design for functional metal-organic frameworks, Chem. Soc. Rev. 41 (2012) 1088-1110.

[2] H.C. Zhou, J.R. Long, O.M. Yaghi, Introduction to metal-organic frameworks, Chem. Rev. 112 (2012) 673-674.

[3] S. Kitagawa, S. Noro, T. Nakamura, Pore surface engineering of microporous coordination polymers, Chem. Commun. (2006) 701-707.

[4] D. Maspoch, D. Ruiz-Molina, J. Veciana, Old materials with new tricks: multifunctional open-framework materials, Chem. Soc. Rev. 36 (2007) 770-818.

[5] J.J.T. Perry, J.A. Perman, M.J. Zaworotko, Design and synthesis of metal-organic frameworks using metal-organic polyhedra as supermolecular building blocks, Chem. Soc. Rev. 38 (2009) 1400-1417.

[6] R.B. Getman, Y.S. Bae, C.E. Wilmer, R.Q. Snurr, Review and analysis of molecular simulations of methane, hydrogen, and acetylene storage in metal-organic frameworks, Chem. Rev. 112 (2012) 703-723.

[7] H.B. Tanh Jeazet, C. Staudt, C. Janiak, Metal-organic frameworks in mixed-matrix membranes for gas separation, Dalton Trans. 41 (2012) 14003-14027.

[8] J. Sculley, D. Yuan, H.-C. Zhou, The current status of hydrogen storage in metalorganic frameworks - updated, Energy Environ. Sci., 4 (2011) 2721-2735.

[9] M. Yoon, R. Srirambalaji, K. Kim, Homochiral metal-organic frameworks for 
asymmetric heterogeneous catalysis, Chem. Rev. 112 (2012) 1196-1231.

[10] Z. Yin, Q.X. Wang, M.H. Zeng, Iodine release and recovery, influence of polyiodide anions on electrical conductivity and nonlinear optical activity in an interdigitated and interpenetrated bipillared-bilayer metal-organic framework, J. Am. Chem. Soc. 134 (2012) 4857-4863.

[11] L. Hashemi, A. Morsali, Microwave assisted synthesis of a new lead(ii) porous threedimensional coordination polymer: study of nanostructured size effect on high iodide adsorption affinity, Cryst. Eng. Comm. 14 (2012) 779-781.

[12] S. Qiu, G. Zhu, Molecular engineering for synthesizing novel structures of metalorganic frameworks with multifunctional properties, Coord. Chem. Rev. 253 (2009) 2891-2911.

[13] D. Zacher, O. Shekhah, C. Woll, R.A. Fischer, Thin films of metal-organic frameworks, Chem. Soc. Rev. 38 (2009) 1418-1429.

[14] M. Meilikhov, K. Yusenko, E. Schollmeyer, C. Mayer, H.-J. Buschmann, R.A. Fischer, Stepwise deposition of metal organic frameworks on flexible synthetic polymer surfaces, Dalton Trans. 40 (2011) 4838-4841.

[15] D. Zacher, K. Yusenko, A. Betard, S. Henke, M. Molon, T. Ladnorg, O. Shekhah, B. Schupbach, T. Arcos, M. Krasnopolski, M. Meilikhov, J. Winter, A. Terfort, C. Woll, R.A. Fischer, Liquid-phase epitaxy of multicomponent layer-based Porous coordination polymer thin films of $[\mathrm{M}(\mathrm{L})(\mathrm{P}) 0.5]$ type: importance of deposition sequence on the oriented growth, Chem. Eur. J. 17 (2011) 1448-1455.

[16] J.K. Yuan, X.G. Liu, O. Akbulut, J.Q. Hu, S.L. Suib, J. Kong, F. Stellacci, Superwetting nanowire membranes for selective absorption, Nanotechnol. 3 (2008) $332-336$.

[17] A.R. Abbasi, K. Akhbari, A. Morsali, Dense coating of surface mounted CuBTC 
metal-organic framework nanostructures on silk fibers, prepared by layer-by-layer method under ultrasound irradiation with antibacterial activity, Ultrason. Sonochem. 19 (2012) 846-852.

[18] M. Y. Masoomi, K. C. Stylianou, A. Morsali, P. Retailleau, D. Maspoch, Selective $\mathrm{CO}_{2}$ capture in metal-organic frameworks with azine-functionalized pores generated by mechanosynthesis, Cryst. Growth Des. 14 (2014) 2092-2096.

[19] J. Aizenberg, Crystallization in Patterns: A Bio-Inspired Approach, Adv. Mater. 16 (2004) 1295-1302.

[20] D. Zacher, A. Baunemann, S. Hermes, R.A. Fischer, Deposition of microcrystalline $\left[\mathrm{Cu}_{3}(\mathrm{btc})_{2}\right]$ and $\left[\mathrm{Zn}_{2}(\mathrm{bdc})_{2}(\mathrm{dabco})\right]$ at alumina and silica surfaces modified with patterned self assembled organic monolayers: evidence of surface selective and oriented growth, J. Mater. Chem. 17 (2007) 2785-2792.

[21] J. Gascon, S. Aguado, F. Kapteijn, Manufacture of dense coatings of $\mathrm{Cu}_{3}(\mathrm{BTC})_{2}$ (HKUST-1) on $\alpha$-alumina, Micropor. Mesopor. Mater. 113 (2008) 132-138.

[22] A.M.B. Furtado, J. Liu, Y. Wang, M.D. LeVan, Mesoporous silica-metal organic composite: synthesis, characterization, and ammonia adsorption, J. Mater. Chem. 21 (2011) 6698-6706.

[23] Mercury 1.4.1, Copyright Cambridge Crystallographic Data Centre, 12 Union Road, Cambridge, CB2 1EZ, UK, 2001-2005.

[24] A.R. Kennedy, K.G. Brown, D. Graham, J.B. Kirkhouse, M. Kittner, C. Major, C.J. McHugh, P. Murdoch, W.E. Smith, Chromophore containing bipyridyl ligands. Part 1: supramolecular solid-state structure of $\mathrm{Ag}(\mathrm{I})$ complexes, New J. Chem. 29 (2005) 826-832.

[25] M. Meilikhov, K. Yusenko, E. Schollmeyer, C. Mayer, H-J. Buschmann, R.A. 
Fischer, Stepwise deposition of metal organic frameworks on flexible synthetic polymer surfaces, Dalton Trans. 40 (2011) 4838-4841.

[26] R. Moosavi, A.R. Abbasi, M. Yousefi, A. Ramazani, A. Morsali, Ultrasound-assisted coating of polyester fiber with silver bromide nanoparticles, Ultrason. Sonochem. 19 (2012) 1221-1226.

[27] M.D. Luque de Castro, F. Priego-Capote, Ultrasound-assisted crystallization (sonocrystallization), Ultrason. Sonochem. 14 (2007) 717-724.

[28] A.R. Abbasi, A. Morsali, Formation of silver iodide nanoparticles on silk fiber by means of ultrasonic irradiation, Ultrason. Sonochem. 17 (2010) 704-710.

[29] E. Guzmán, H. Ritacco, J.E.F. Rubio, R.G. Rubio, F. Ortega, Salt-induced changes in the growth of polyelectrolyte layers of poly(diallyl dimethylammonium chloride) and poly(4-styrene sulfonate of sodium), Soft Matter 5 (2009) 2130-2142.

[30] A. Yu. Grosberg, T. T. Nguyen, B. I. Shklovskii, Colloquium: the physics of charge inversion in chemical and biological systems, Rev. Mod. Phys. 74 (2002) 329-345.

[31] A.R. Abbasi, A. Morsali, Synthesis and properties of silk yarn containing Ag nanoparticles under ultrasound irradiation, Ultrason. Sonochem. 18 (2011) 282-287.

[32] P. Horcajada, C. Serre, G. Maurin, N.A. Ramsahye, F. Balas, M. Vallet-Regi, M. Sebban, F. Taulelle, G. Ferey, Flexible porous metal-organic frameworks for a controlled drug delivery, J. Am. Chem. Soc. 130 (2008) 6774-6780. 


\section{Figure caption}

Figure 1. (A) Schematic representation of the formation mechanism of TMU-4 and TMU-5@silk fiber: (a) water circulation, (b) double jacketed vessel, (c) ultrasound bath and (d) silk yarn. (B) Layers of $\mathrm{Zn}$ (II)-oba pillared by 4-bpdb (TMU-4) and 4-bpdh (TMU-5).

Figure 2. SEM image of the pristine silk fiber (A), SEM photographs and the corresponding particle size distribution histograms of TMU-5@silk after applying 4 (B) and 15 deposition cycles under ultrasound irradiation (C), TMU4@silk after applying 4 (D) and 15 deposition cycles under ultrasound irradiation (E).

Figure 3. SEM photograph and the corresponding particle size distribution histogram of TMU-4@silk (A) and TMU-5@silk (B) after applying 4 deposition cycles without sonication.

Figure 4. Temporal evolution of UV/vis absorption spectra for the loading of morphine from TMU-4@silk (A) and TMU-5@ silk (B).

Figure 5. Temporal evolution of UV/vis absorption spectra for the delivery of morphine from TMU-4@silk (A) and TMU-5@silk (B) containing morphine.

Figure 6. Morphine adsorption (A) and release (B) from TMU-4@ silk and TMU-5@ silk containing morphine. 


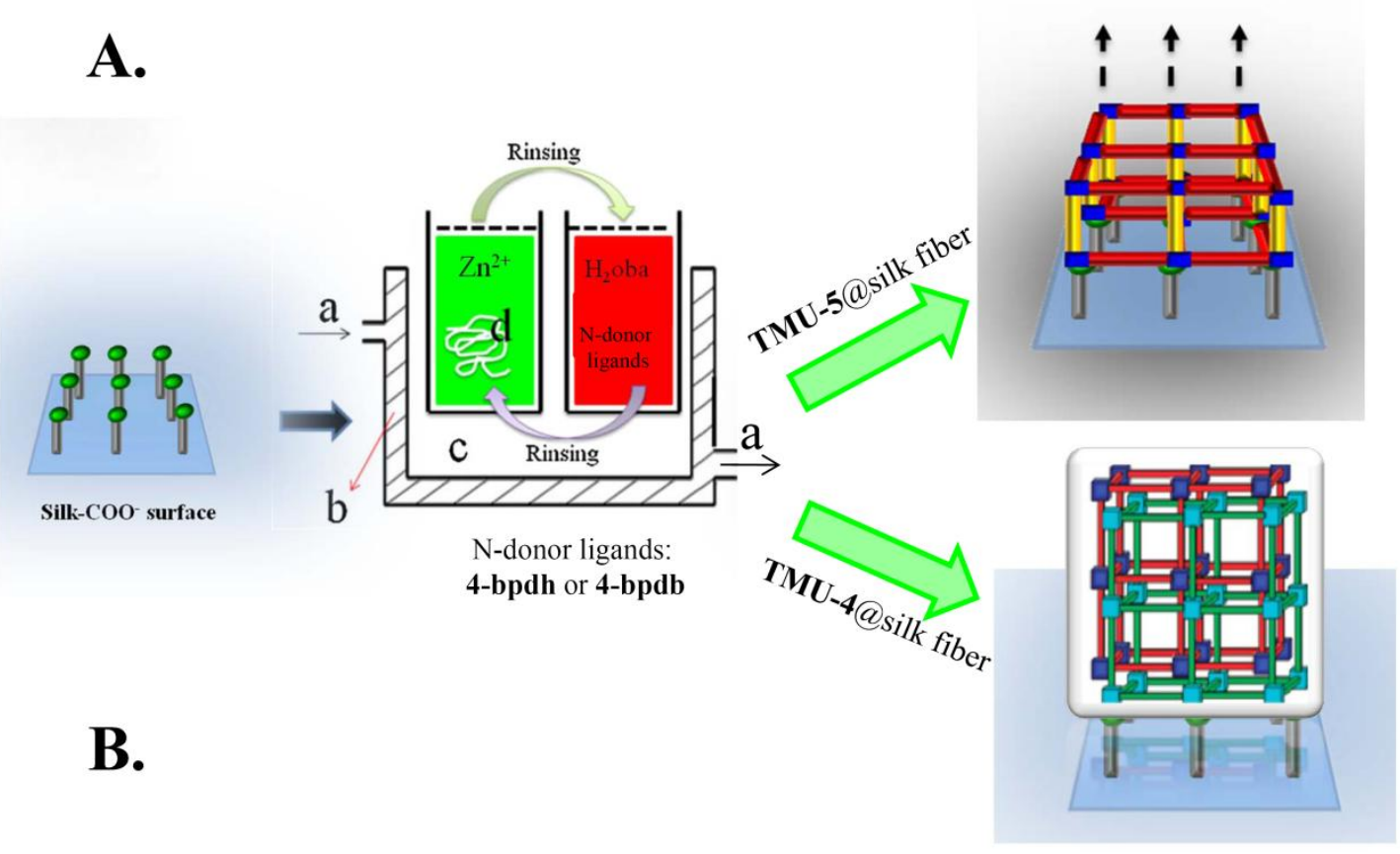

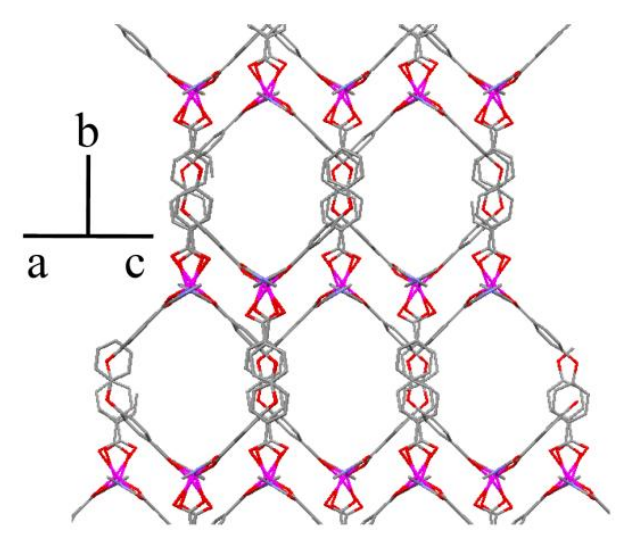

TMU-4

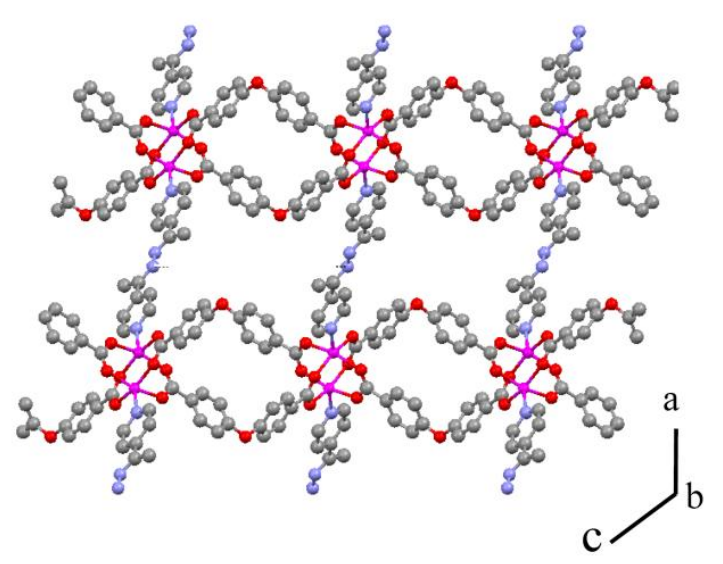

TMU-5

Figure 1. 

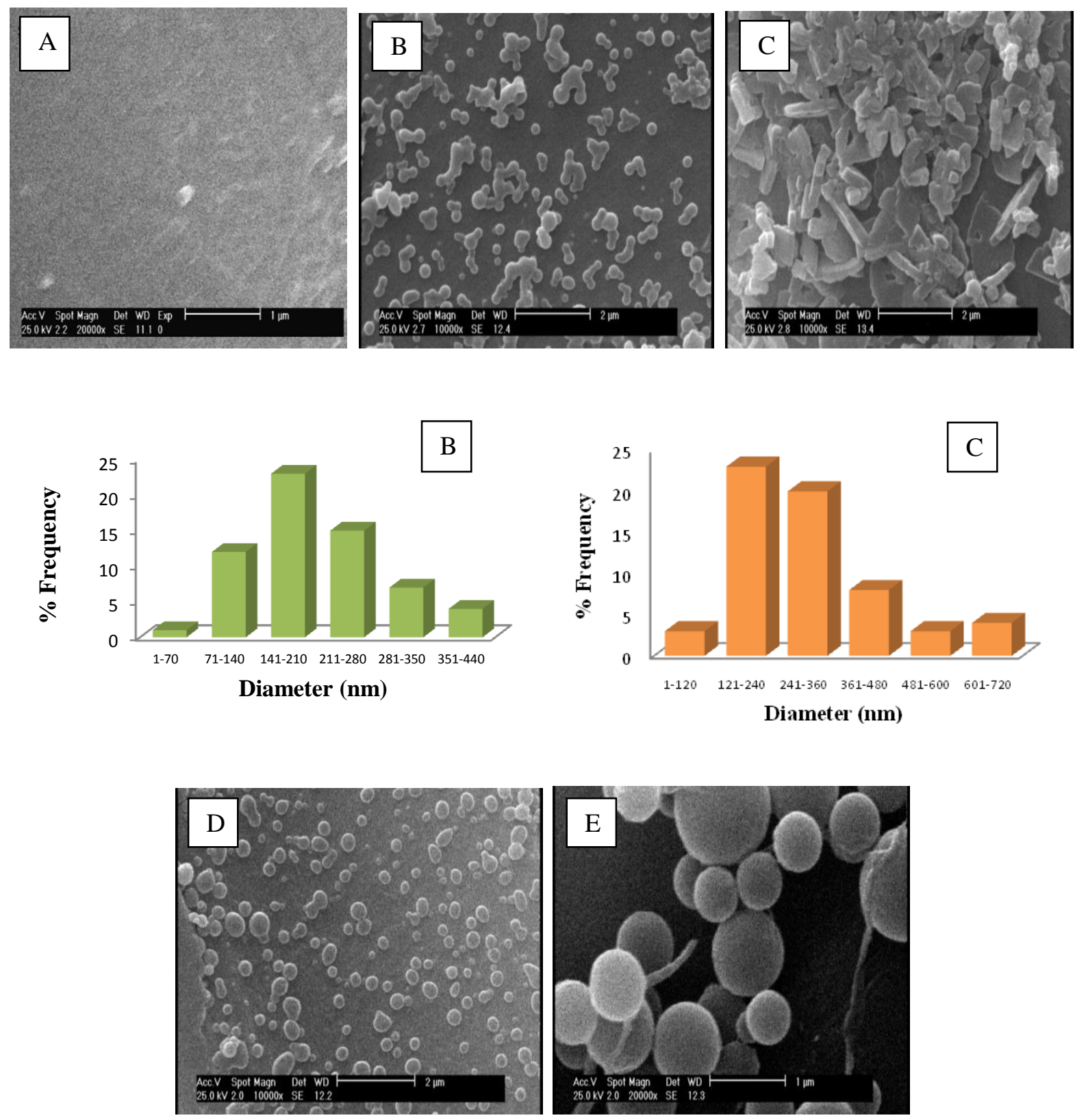

Figure 2. 

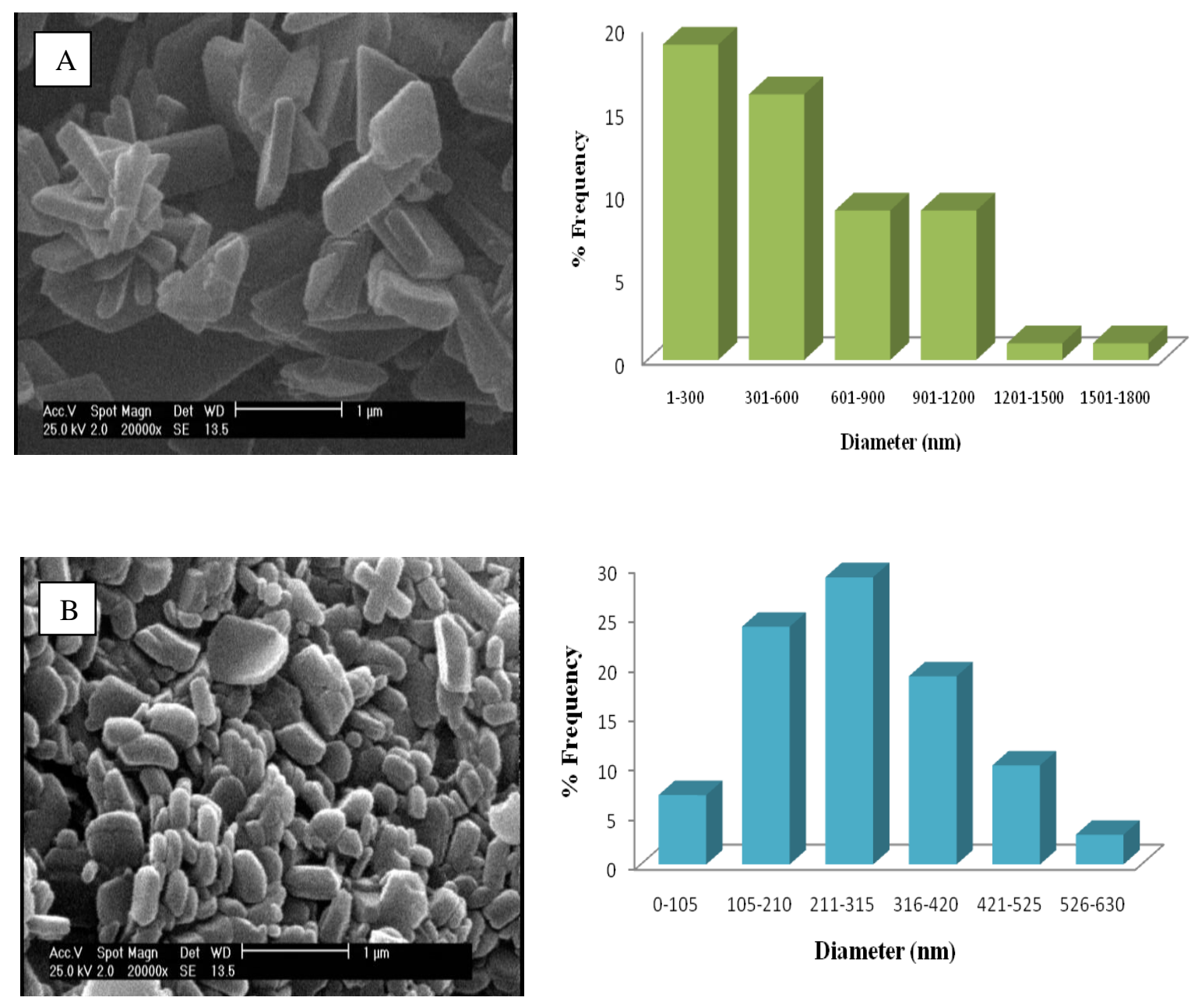

Figure 3. 

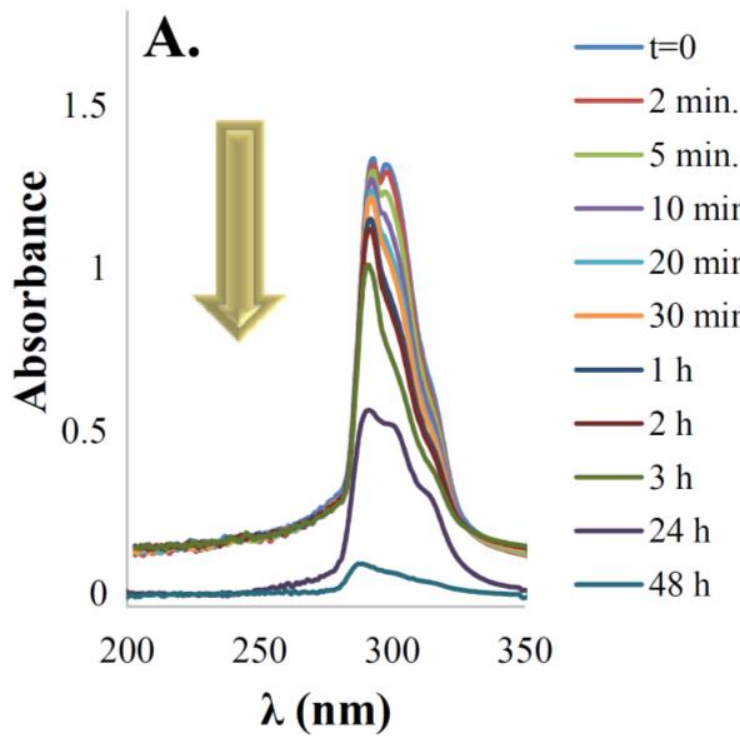

$-5 \mathrm{~min}$.

$-10 \mathrm{~min}$.

$-20 \mathrm{~min}$.

$-30 \mathrm{~min}$.

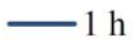

$-2 \mathrm{~h}$

$3 \mathrm{~h}$

$-24 \mathrm{~h}$

$-48 \mathrm{~h}$
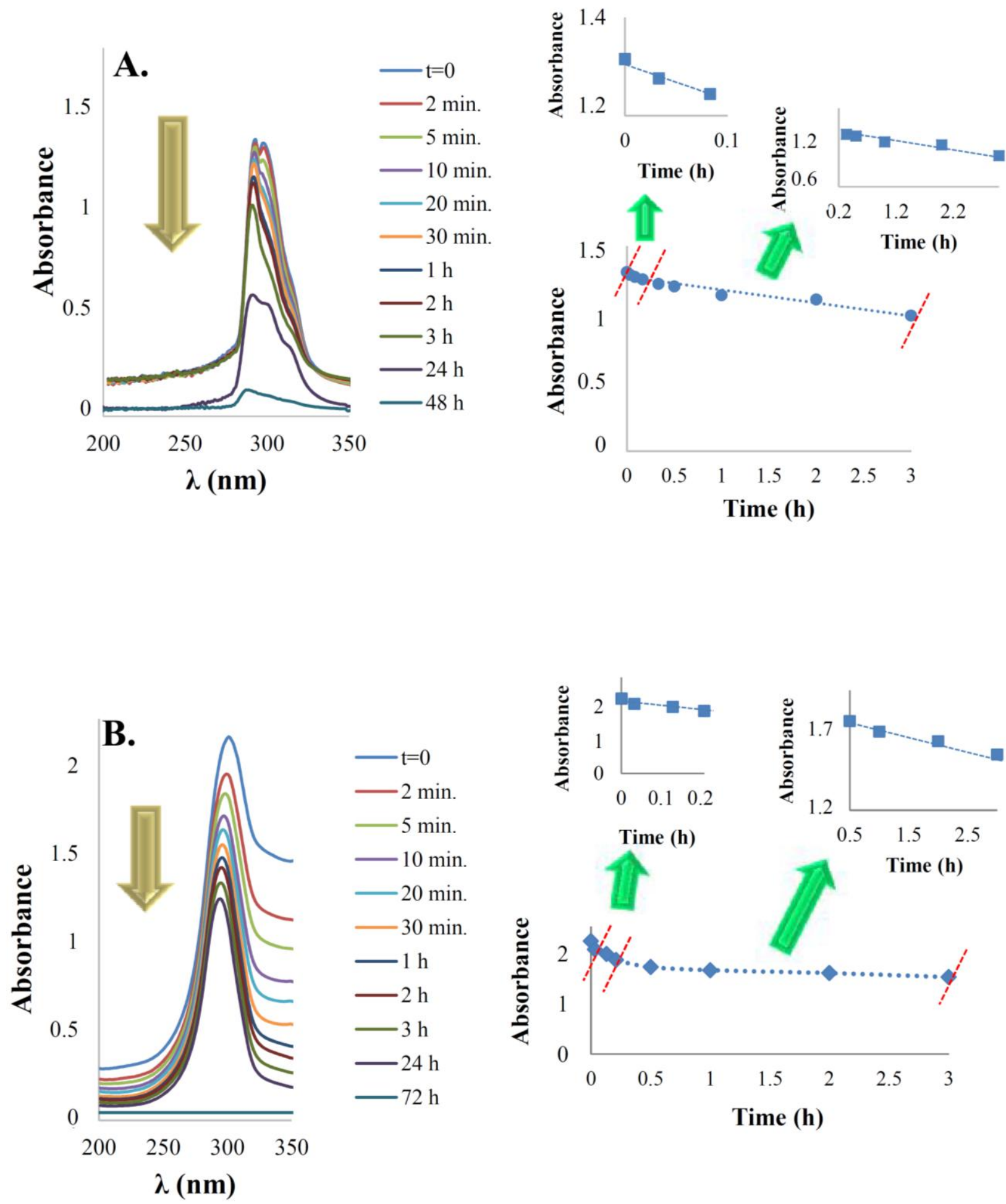

$-\mathrm{t}=0$
$-2 \mathrm{~min}$.

$-5 \mathrm{~min}$.

$-10 \mathrm{~min}$.

$-20 \mathrm{~min}$.

$-30 \mathrm{~min}$.

$-1 \mathrm{~h}$

$-2 \mathrm{~h}$

$-3 \mathrm{~h}$

$-24 \mathrm{~h}$

$-72 \mathrm{~h}$
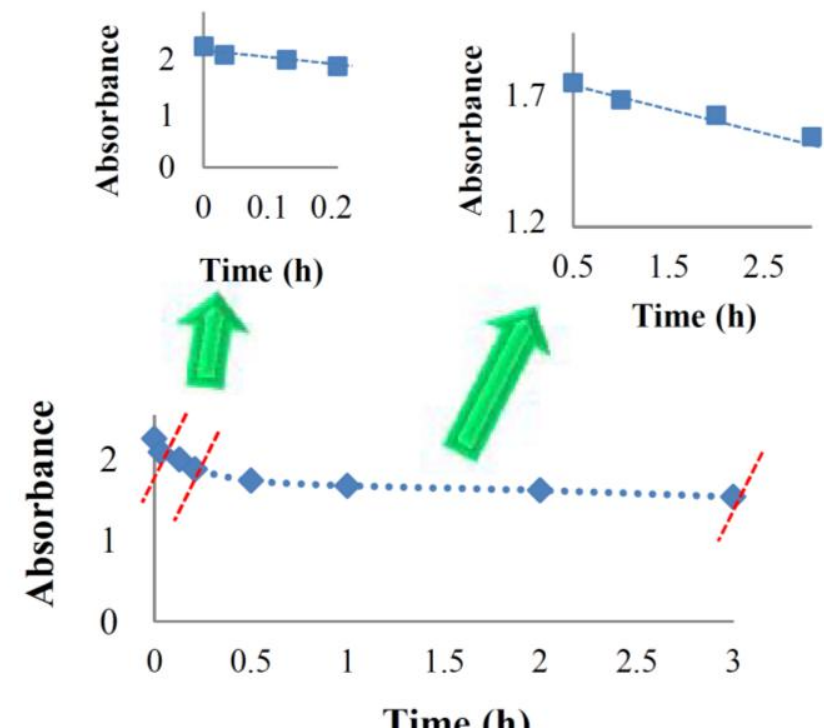

Time (h)

Figure 4. 

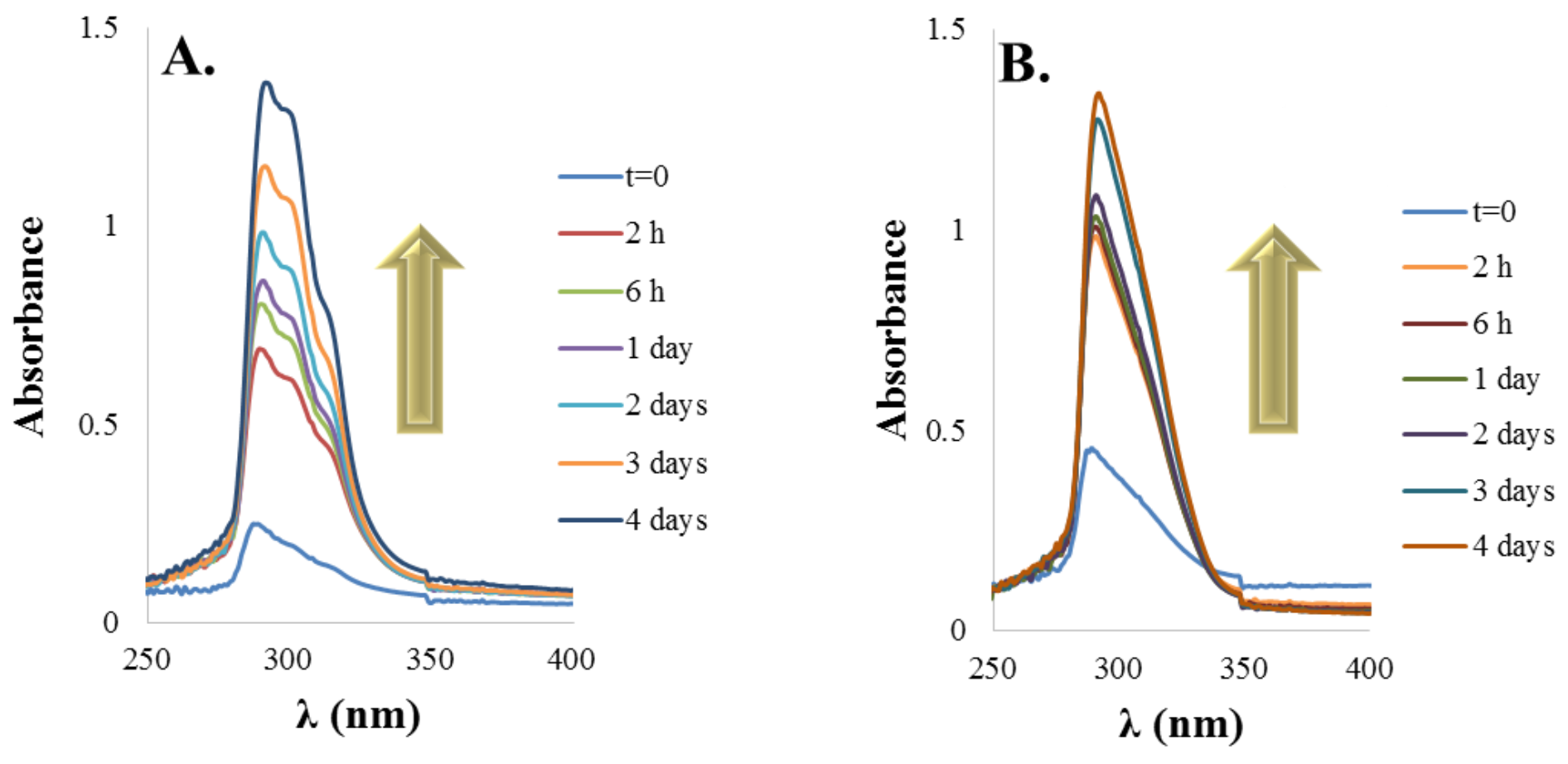

Figure 5. 


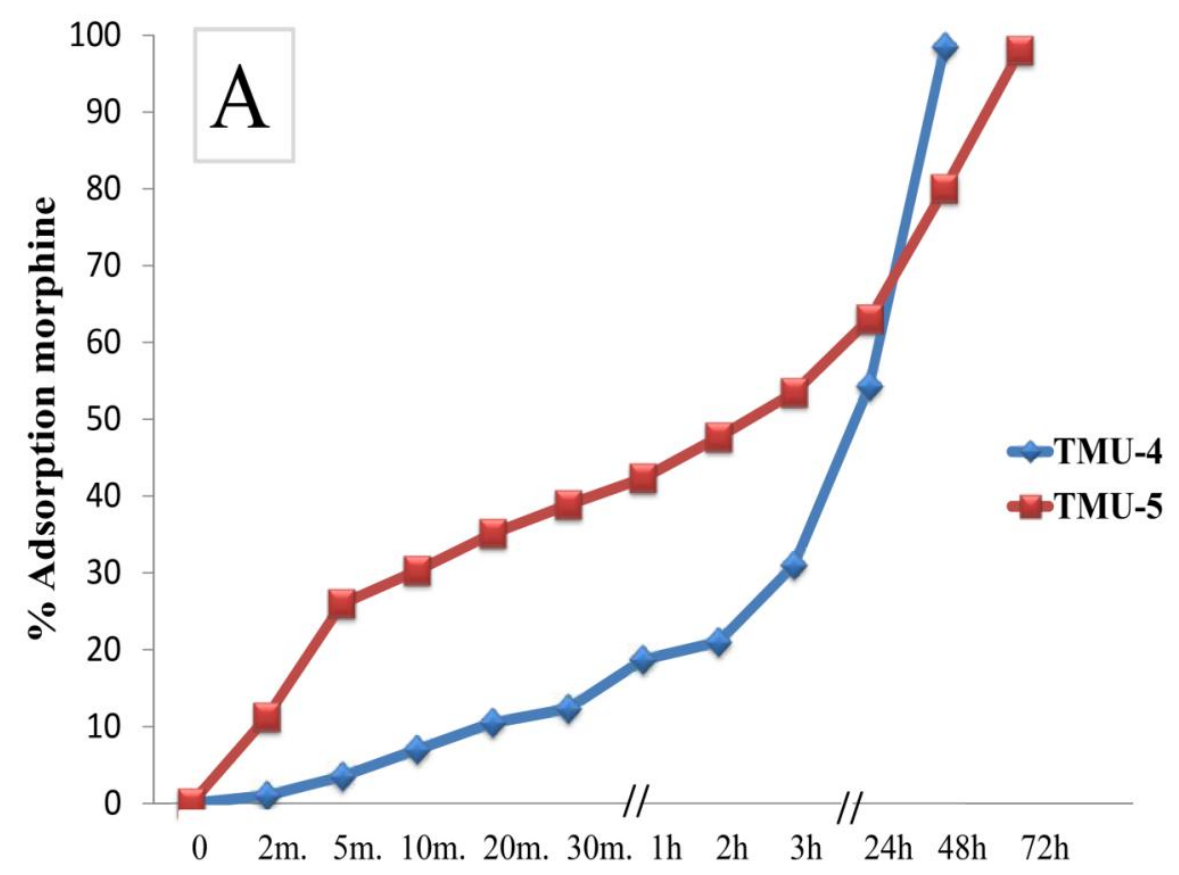

Time

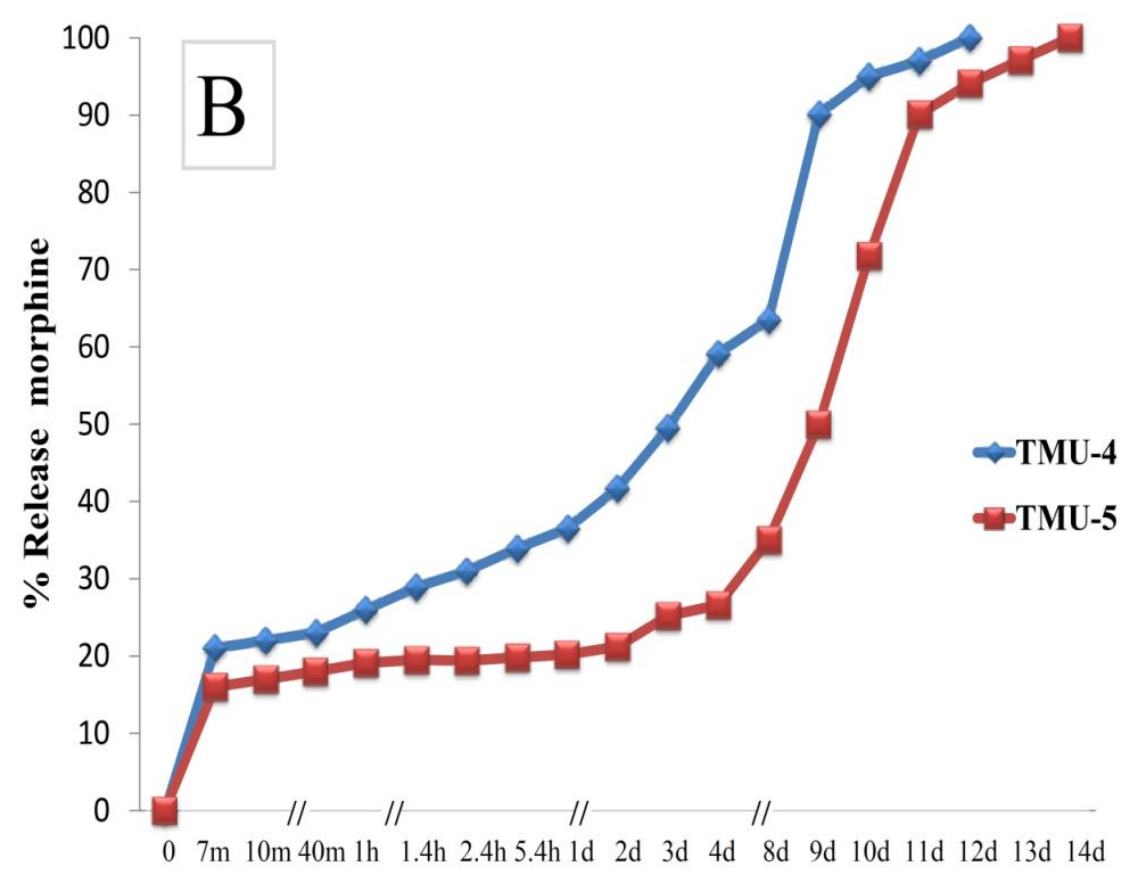

Time

Figure 6. 


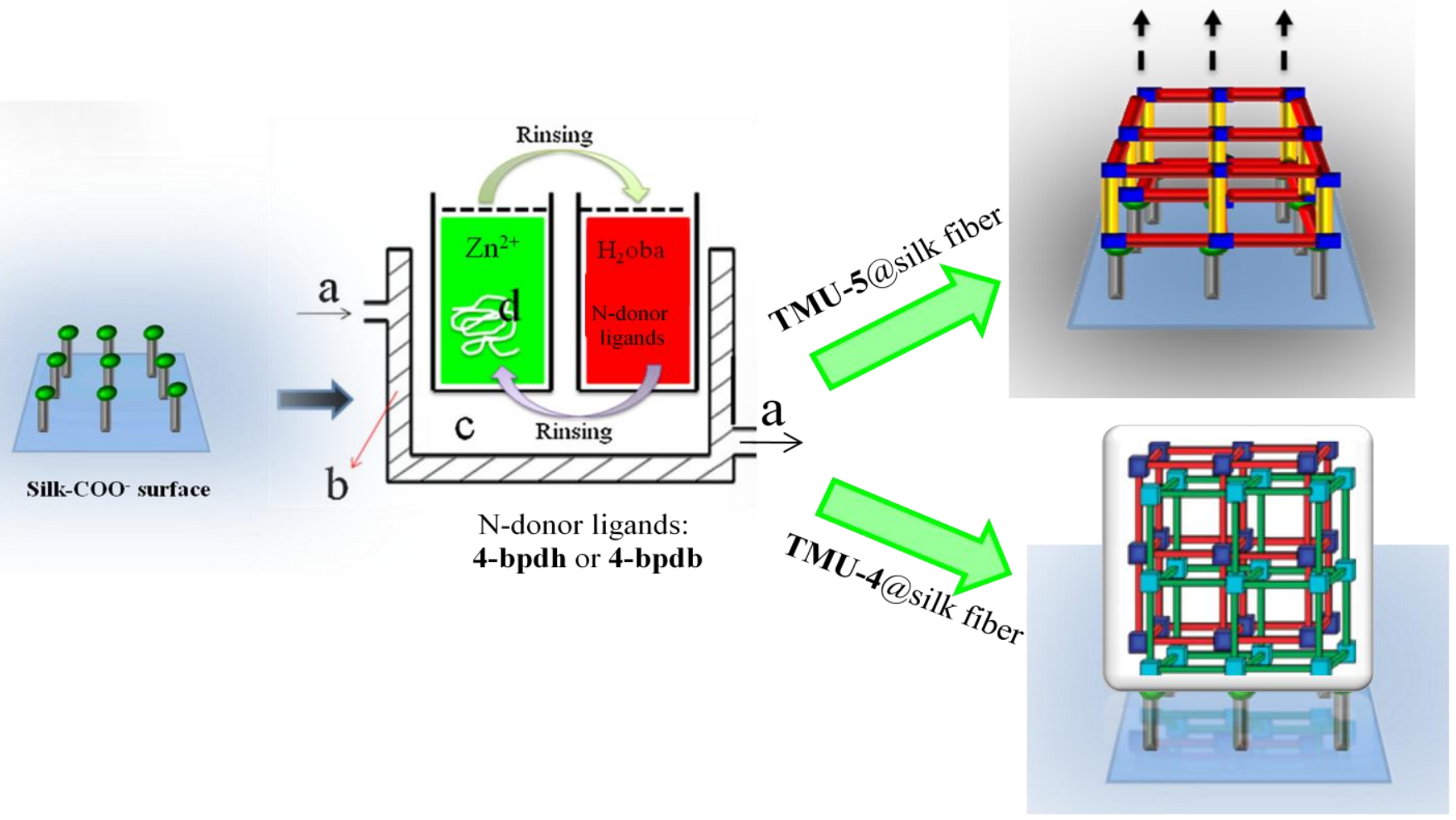

The formation mechanisms of TMU-4 and TMU-5 metal-organic frameworks upon silk fiber under ultrasound irradiation. 